\title{
Correction to: Redescription of the bigeye chimaera, Hydrolagus macrophthalmus de Buen, 1959 (Chondrichthyes: Chimaeriformes), with a genetic characterization of the species
}

\author{
Matthew Jew ${ }^{1} \cdot$ David A. Ebert ${ }^{1,2,3} \cdot$ Jenny M. Kemper ${ }^{4} \cdot$ Kristin Walovich $^{1} \cdot$ Kimberly L. Quaranta $^{1}$
}

Published online: 24 July 2019

(C) Senckenberg Gesellschaft für Naturforschung 2019

\section{Correction to: Marine Biodiversity}

https://doi.org/10.1007/s12526-018-0911-8

The authors regret to have published the wrong Fig. 4 of this manuscript. The correct Fig. 4 is included here. This mistake does not affect the concept or any other information provided in other parts. In addition, the results and conclusions of the paper also remain valid.

The authors sincerely apologize for this mistake.

The online version of the original article can be found at https://doi.org/ 10.1007/s12526-018-0911-8

Matthew Jew

mjew@mlml.calstate.edu

1 Moss LandingMarine Laboratories, 8272Moss Landing Road, Moss Landing, CA, USA

2 Department of Ichthyology, California Academy of Sciences, 55 Music Concourse Drive, San Francisco, CA 94118, USA

3 South African Institute for Aquatic Biodiversity, Private Bag 1015, Grahamstown 6140, South Africa

4 Hollings Marine Laboratory, Medical University of South Carolina, 331 Fort Johnson Road, Charleston, SC 29412, USA 
Fig. 4 Maximum likelihood tree topology using general-time reversible substitution model and gamma distribution on aligned nucleotide NADH2 sequences for Hydrolagus macrophthalmus and comparativematerial. Outgroup is represented by Harriotta raleighana. Bootstrap support values are displayed on major nodes. GenBank accession numbers follow species name
Hydrolagus macrophthalmus Eastern Pacific Panama MG356492

Hydrolagus macrophthalmus Eastern Pacific Panama MG356496

Hydrolagus macrophthalmus Eastern Pacific Panama MG356501

Hydrolagus macrophthalmus Eastern Pacific El Salvador MG356494

Hydrolagus macrophthalmus Eastem Pacific Panama MG356497

Hydrolagus macrophthalmus Eastern Pacific Panama MG356495

Hydrolagus macrophthalmus Eastern Pacific Panama MG356499

Hydrolagus macrophthalmus Eastern Pacific Panama MG356493

100

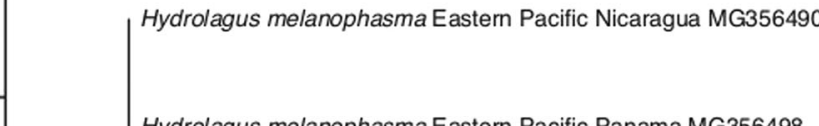

Hydrolagus melanophasma Eastern Pacific Panama MG356498

98

Hydrolagus melanophasma Eastern Pacific Nicaragua MG356500

Hydrolagus melanophasma Eastem Pacific Nicaragua MG356491

95

Hydrolagus trolli New Zealand KU934288

Hydrolagus trolli New Zealand KU934293

100

Hydrolagus trolli Victoria KU934289

Harriotta raleighana North Atlantic KU163643

-0.01 substitutions/site

Publisher's note Springer Nature remains neutral with regard to jurisdictional claims in published maps and institutional affiliations. 